\title{
Medullary Bone
}

National Cancer Institute

\section{Source}

National Cancer Institute. Medullary Bone. NCI Thesaurus. Code C53466.

The central and spongy portion of the bone. 